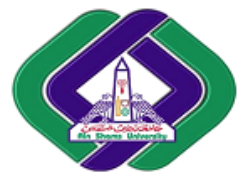

\title{
Exploring and Measuring the Key Performance Indicators in Higher Education Institutions
}

\author{
Mohammed Badawy \\ Dept. of Information Systems and \\ Technology, Institute of Statistical \\ Studies and Research (ISSR) \\ Cairo University, Giza, Egypt \\ mbadawy@live.com
}

A. A. Abd El-Aziz

Dept. of Information System, College of Computer and Information Sciences, Jouf University, Kingdom of Saudi Arabia

a.ahmed@cu.edu.eg
Hesham Hefny

Dept. of Information Systems and Technology, Institute of Statistical Studies and Research (ISSR) Cairo University, Giza, Egypt

hehefny@hotmail.com

\begin{abstract}
To enhance the quality of higher education institutions (HEIs), key performance indicators (KPIs) must be explored and measured. KPIs deems as a measurable value which explains the effectiveness of an institution and how it is achieving key objectives. Institutions use KPIs for ensuring that they are going on the right way or not. Many of the indicators for HEIs have been developed before, but the main question is how to choose the indicators that fit the institution for achieving goals and how to measure these indicators. This paper provides a model to explore and measure KPIs using text mining and feature extraction technique and measure indicators automatically rather than traditional methods of exploring based a questionnaire, we measure KPIs to know the impact of exploring these KPIs on the overall performance in HEIs. The data for the present study was collected by the Institute of Statistical Study and Research (ISSR) in Cairo University, and the impact of KPIs on the overall performance of the institution was evaluated. This study adopted pre-processing techniques and keyword extraction using text mining tool (RapidMiner) to conduct the research.
\end{abstract}

Keywords: Key Performance Indicator (KPI); Text Mining; Term Frequency; Tokenization; Stop Words; Stemming.

\section{Introduction}

KPIs are the most comprehensive goals of the organizations which guide the managers`activities for making them obtainable. They are very important and they are considered of significance in quality improvement and objective attainment. There are a lot of studies on the position of key performance indicators and some of them appear as follows. Hubert (1984) postulated that without a general conception of past events, no constant change or improvement will happen. There will be no constant change and improvement in the growing of the university's quality. Of course without performance evaluation based on key factors and indicators, because the major mission or the main job of management is evaluating the performance is to apply and gain the main strategies of the organization, it is found that evaluating performance is a necessary need for universities. The admission of KPIS is a major step to performance evaluation. Moreover, for choosing the key indicators, one has to consider 
the organization and benefit maker's demands and needs. Then, the key indicators and goals are going to be settled and recognized. Finally, they must be used in a convenient model of performance evaluation [1].

Assessment is typically viewed as a tool of diagnosing to be used in measuring the success of the institutions in fulfilling its educational goals that improve student performance in education. Before taking the decision of teaching, first we need an obvious idea of what is wanted from our students to study and know. Obviously must know how to stipulate what is needed to be understood from each subject. Then, we must explain our objectives in terms to make students able to demonstrate their understanding. This paper is an extension of my research [2].

\section{A. Higher Education Learning Outcomes}

Developing learning outcomes are a comparatively new phenomenon in higher education which has lately increased interest. We see that the Higher education learning outcomes have attracted the interest of policy makers, agencies of quality assurance, and university administrators among others and involved university teachers in formulating the expected or required 'outcome' or result of studying programs. The higher education learning outcomes can refer to two main things: first they can identify and clarify the content of what students have studied and learnt after the course or the degree (prescribed outcomes); second, they can refer to the measurement of what students have learnt (achieved outcomes) [3]. Intended learning outcomes for higher education are four domains, which cover the expertise of understanding, intellectual skills, professional skill, general transferable skills, and practical skills [4].

\section{B. Text Mining}

Text mining is a process for extracting significant patterns for exploring the knowledge from textual data sources. Text mining is a multi-disciplinary field based on information retrieval, data mining, machine learning, statistics, and computational linguistics. Figure 1 shows the diagram of text mining and its interaction with other fields. Text mining deals with natural language text which is stored in an unstructured format. So Text mining techniques are applied in industry, academia, internet and other fields. Application areas like search engines, customer relationship management system, filter emails, fraud detection, and social media analytics use text mining for feature extraction and predictive...etc. [5]. 


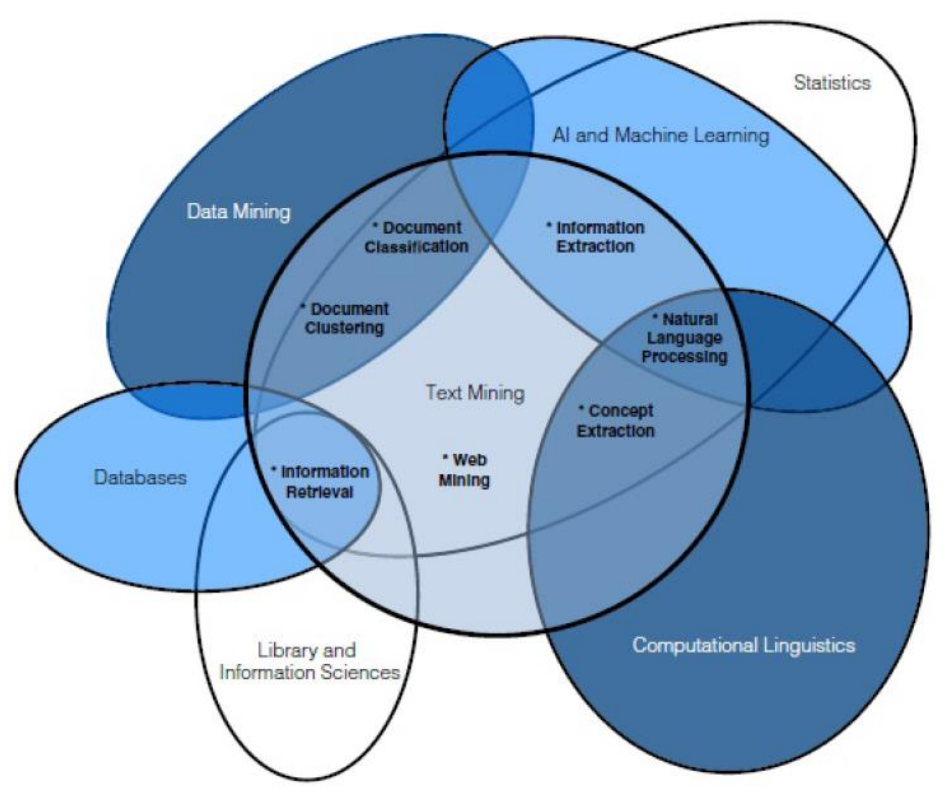

Figure. 1: Text mining interaction with other fields [5]

There are needs to provide processes that help in exploring KPIs, the proposed model attempted to answer the following questions:

1- What are the processes being used in exploring KPIs for academic program and students?

2- How can institutions measuring KPIs to ensure their growth and competitive advantage?

\section{Literature Review}

In [6], the authors adopted Fuzzy Delphi method to set up KPIs in Taiwan basic education when he collected 12 expert scholars' opinions on a questionnaire. Among the 37 indicators constructed and built in this study, the top five understood by the experts as most important had Gi values $>8.75$. From the highest to the lowest or, from top to bottom, these indicators were "Learning achievement performance: Student learning performance in various learning domains", "Parental satisfaction: It is parents satisfaction toward school", "Physical fitness performance: Degree of student performance beyond standard criteria for physical fitness tests", "3-3-2 School reputation \fame: Degree of high regard of community (society) for the school", and "School culture: Degree of warmth and care exhibited or displayed by school staff of personnel"'.

In [1], the author introduced a study to find KPIs and to offer a conceptual structure to estimate the universities' performance according to KPIs. Factor analysis and (Kaiser-Meyer-Olkin Sampling Measure) KMO were used for evaluating the data. SPSS and Alpha Koronbach were used to test the inner validity. In the end, it is based on the findings, the researcher portraits 151 indicators and 3 conceptual frameworks. The method of research is attachment or correlation. When the related literature is reviewed, KPIs and the variables concerning the universities were found. To collect the data the researcher-made questionnaire which was used? SPSS software was used to calculate the internal validity and alpha degree \%938 was measured. The research subjects are all managers of Islamic Azad universities of the region (78 managers) together with some faculty members (242). The researcher 
mentioned that his research faced with some limitations like the shortage of high education experts and the impracticality of regular plans of management in the university area.

In [7], the author provided the university academics' impression of the strengths and weaknesses in the common Performance Indicators used for research and teaching in many Australian universities and four Australian universities were selected for the survey. They used a questionnaire and interview to collect the required data from academic staff. Participation was voluntary and optional, and the participants were assured of confidentiality and exclusiveness of results. Out of the 287 questionnaires which were sent, only 152 were completed and returned. So this represented an answer rate of 53\%. A party of the group (43 academics) was met to interview. The academics in this study were predominantly (mostly) male, in full-time permanent (constant) employment (86\%), did both research and teaching (77\%), were lecturers (Level B), and have been in the current universities for 10 years. The ultimate and most tests of the worth of PIs is the extent and the range to which their progress the quality of the decisions made by the people that were exposed to them, whether this is the government, university management or the individual academic.

In [8], the author presented a model of KPI measurement in HEIs based on a mixture between Analytical Hierarchy Process, trend analysis and comparative data, and classified into academic, research, and supporting KPIs. All KPIs weighed by using Analytic Hierarchy Process. Furthermore, KPIs points are set based on its trend over last three years and its current level compared to benchmark or competitor performances. The suggested model shares to measure and explains the success of institution by using multi dimensions of KPIs. Better performance of HEIs is reflected by the growth of organization results which are demonstrated by current level compared to historical performances; and besides, it appears when comparing between the current level and the level of competitor performances. The author referenced that he adopted on a closed set of KPI so there could be another set not used or undiscovered.

\section{Methodology and Model}

In this study, we provide an idea of how the model is built and the algorithm used to explore the KPIs for institutions using text mining, getting synonyms of words, and keyword extraction technique. The study was conducted in Statistical Studies and Research (ISSR) computer and information science department and the course selected randomly from a list of education courses offered in the second semester 2016 -2017.The tools used in this research were:

- RapidMiner version 5.3.015.

- WordNet version 2.1.

- Microsoft Excel version 2010. 


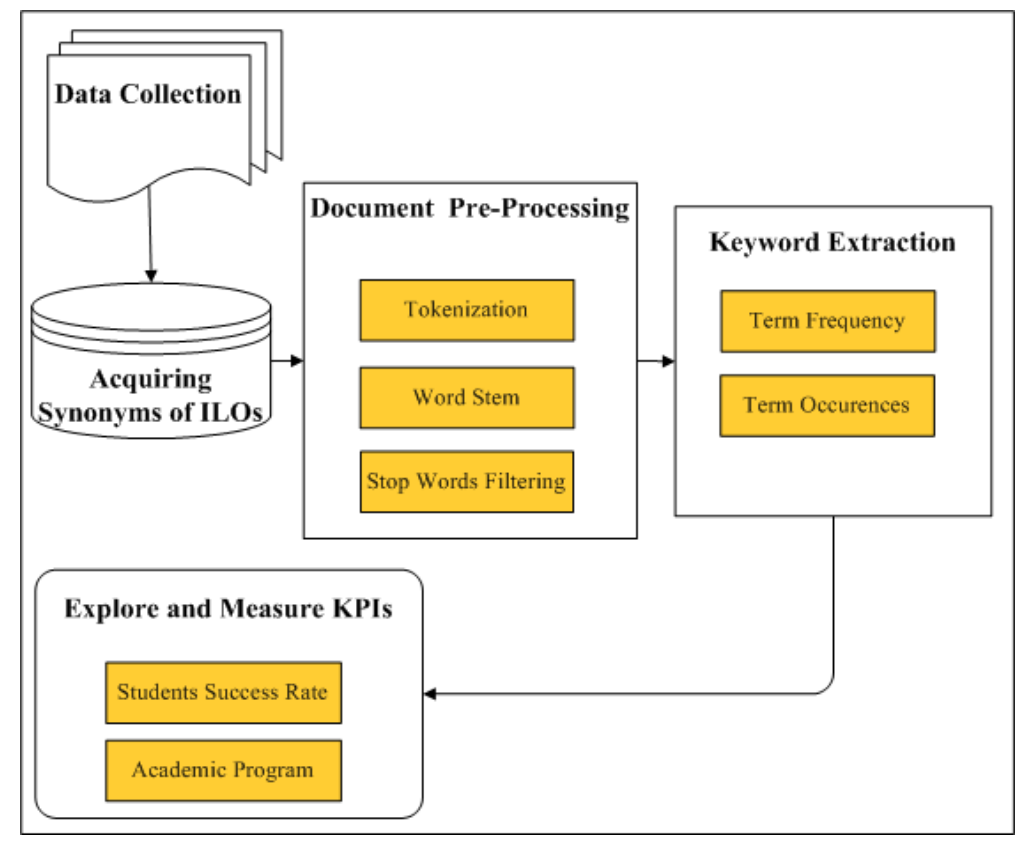

Figure. 2: The Architecture of the Proposed Model

\section{A. Data Collection Phase}

The data were collected as follows:

1. Collecting learning objectives for a Java course of the second semester, the selected Java textbook titled (Java: How to Program, Ninth Edition) which contains 31 chapters. Learning objectives were collected for each chapter stored in a text file to be used it in the next phase.

2. Collecting verbs of ILOs from Information System \& Technology Department, ISSR.

\section{B. Acquire Word Synonyms of Intended Learning Outcomes Phase}

A synonym is a word which can be substituted or replaced with another without important change of meaning. WordNet is a large lexical database (a collection of words) of English used as an information base in automatic text Analysis and artificial intelligence, which consist of many groups of synonym words of the same meaning [9]. In this phase, verbs of ILOs were collected in all domains in addition to obtaining their synonyms and compilation in a separate document for each domain.

\section{Document Pre-Processing Phase}

The data mining tool (RapidMiner) was used to convert the text into a structured representation. That allows users to transform the text into a structured representation. The steps of data pre-processing phase where illustrated in Figure 3 as below: 


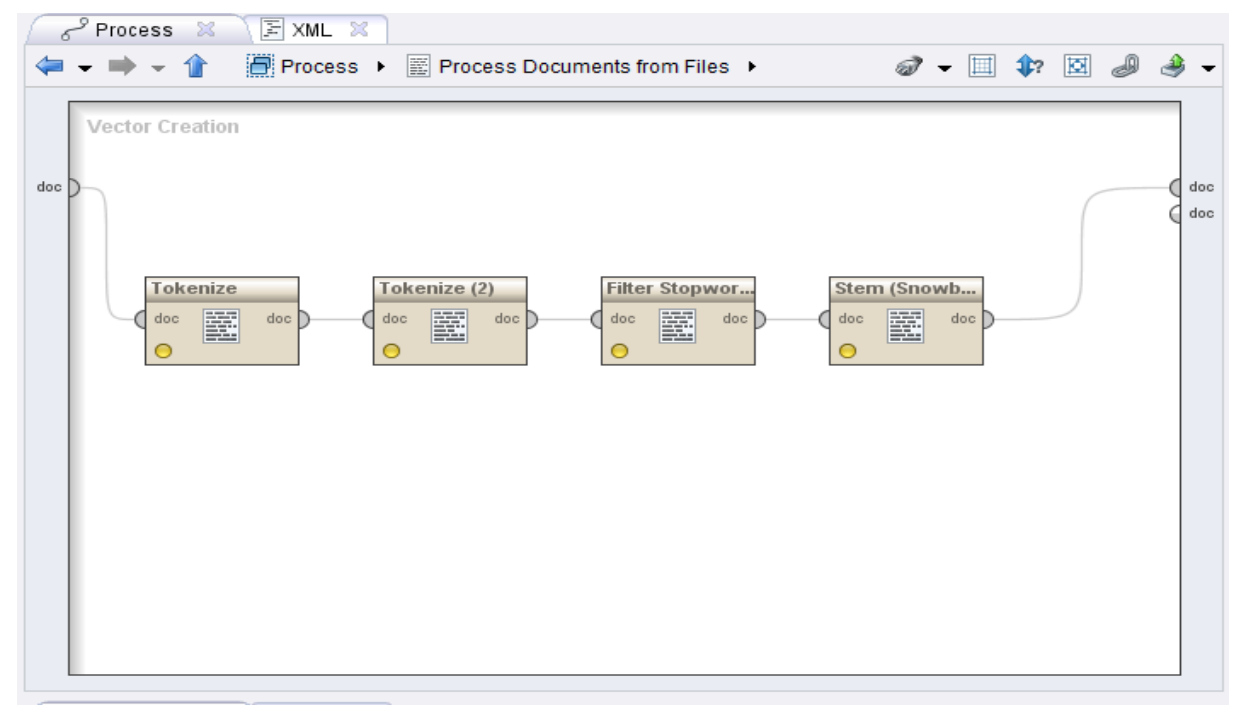

Figure. 3: Flow of a Pre-Processing in RapidMiner

The phase of data Pre-Processing completed in 4 steps as follows:

1. Tokenization: it includes two steps, the first to remove the letters using mode (none letters) and the second step to remove the regular expression which is in the learning objectives of textbook using mode (regular expressions) [-!"\#\$\%\&'()*+,./:;<=>?@ $\left.\left.\backslash[I I I]_{-} \backslash\{\mid\} \sim\right]\right)$.

2. Stop Words Removal: stop words are the much occurred group of words which do not aggregate relevant information. The examples of stop words are (a, an, and, are)... etc.

3. Stemming: it works as dimension reduction. This process designed for decreasing inflected or derived words to their stem (base) form, such as (induct|ed : induct|ion ).

\section{Keyword Extraction Phase}

Keywords are index terms (words) of document that contain most important information [10]. Automatic keyword extraction is the mission to identify a small group of terms, key phrases, keywords, and key segments from a document which describe the sense of the document. Many text mining applications can take advantage of automatic keyword extraction, for example: automatic indexing, automatic filtering, automatic summarization, automatic classification, etc. Thus, keywords extraction is considered the core technology of all automatic processing for documents. Anyway, a large number of documents don't include keywords. Simultaneously, manual assignment of high quality keywords is costly and error prone, and time-consuming [11].

Keyword extraction automatically identifies a group of the terms that best describe the subject of the document. Extracting a small set of units, composed of one or more terms, from a single document is an important problem in Text Mining, Information Retrieval and Natural Language Processing [12].

According to the curriculum of the second semester Java course were selected with a textbook (Java: How to Program [Deitel]) which contains 31 chapters. Term frequency was obtained from the verbs of ILOs in all chapters using RapidMiner tool, as a follow:

\section{D1. Knowledge and Understanding}

The domain of knowledge and understanding are the main information to be gained and the concepts that should be understood from the course. This domain contains 16 verbs according to ILOs of ISSR, after this getting the synonyms of verbs using a lexical database of English (WordNet). Figure 4 
displays the frequency of the verbs for knowledge and understanding with the learning objectives in every chapter of the textbook.

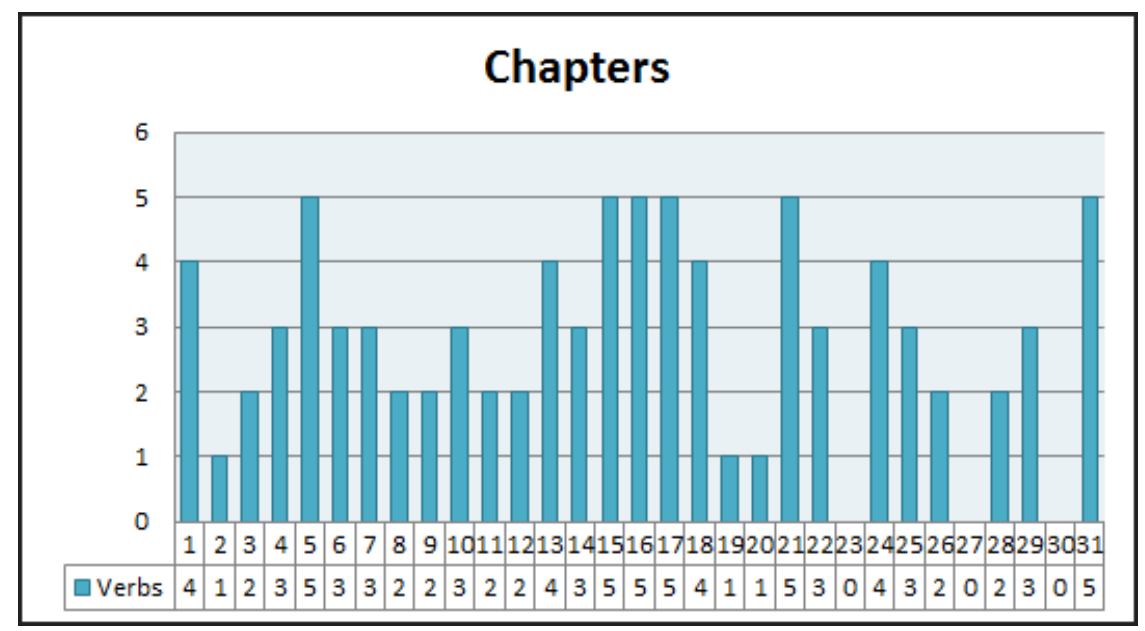

Figure. 4: Knowledge and Understanding

\section{D2. Intellectual Skills}

Refer to analytical and problem solving skills, including the assimilation and capacity of new knowledge, this domain contains 18 verbs according to ILOs of ISSR, after this getting the synonyms of verbs using a lexical database of English (WordNet). Figure 5 displays the frequency of the verbs for intellectual skills with learning objectives in every chapter of the textbook.

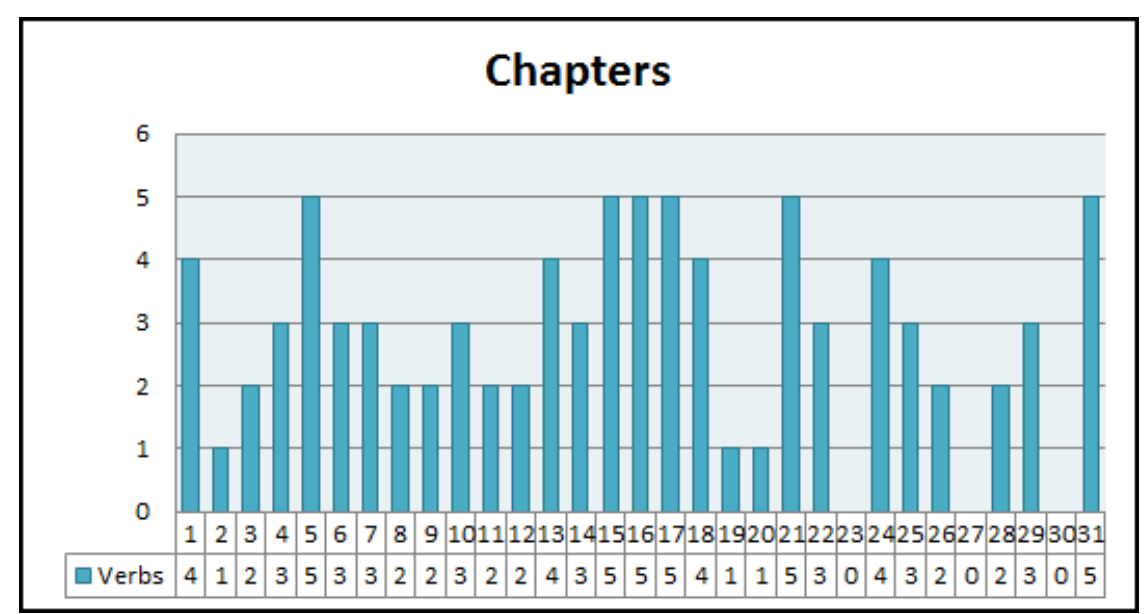

Figure. 5: Intellectual Skills

\section{D3. Professional and Practical Skills}

Professional and practical skills are the ability of the student after completing the course to apply and adopt the topics into professional applications. This domain contains 18 verbs according to ILOs of 
ISSR, after this getting the synonyms of verbs using a lexical database of English (WordNet). Figure 6 displays the frequency of the verbs for professional and practical skills with learning objectives in every chapter of the textbook.

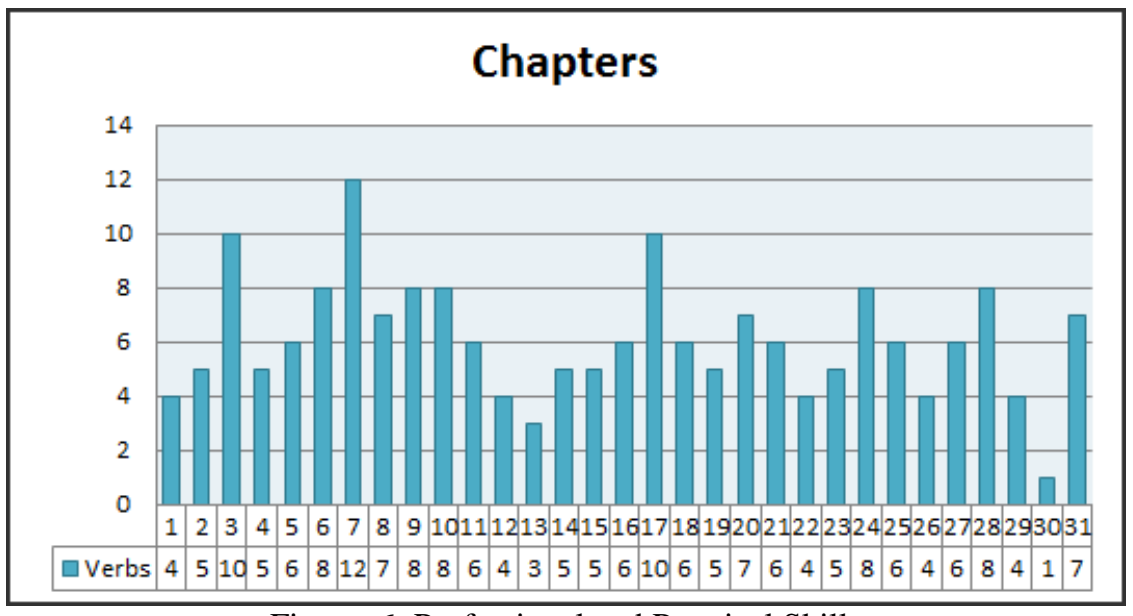

Figure. 6: Professional and Practical Skills

According to this method, after preprocessing and keyword extraction using term frequency and document occurrences and calculating the number of times the verb was appearing in learning objectives of chapters. Figure 7 displays results of match ILOs verbs with learning objectives using word synonyms.

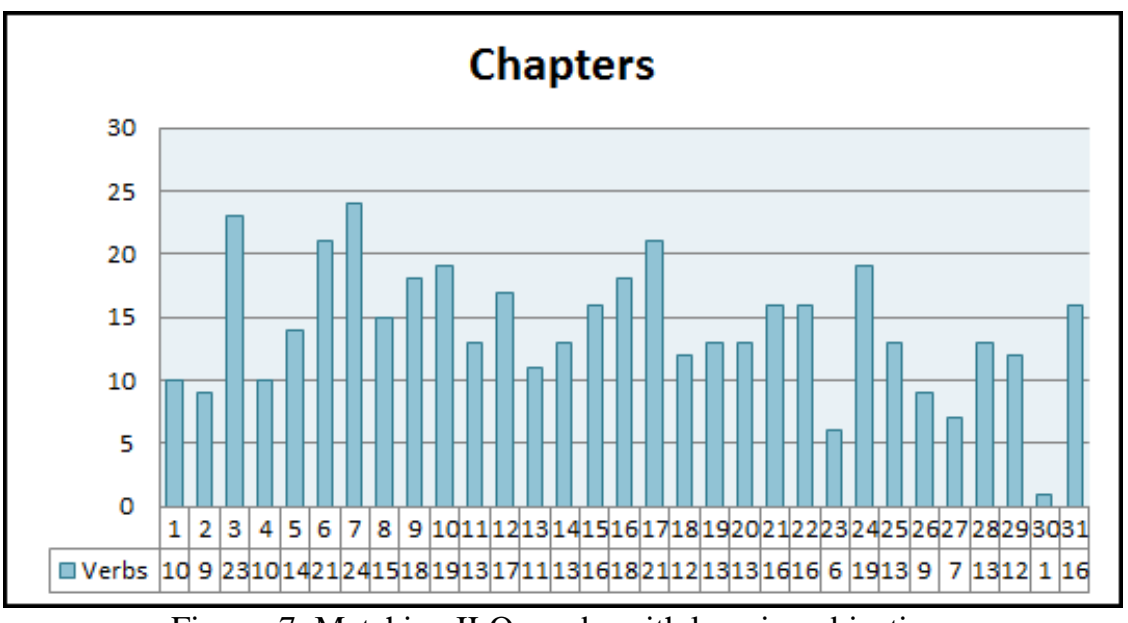

Figure. 7: Matching ILOs verbs with learning objectives

For calculating the rank of the chapters, the frequency of term incidences within a document has frequently been used, Table 1 shows the weight of each chapter according to the rank in which the verbs of ILOs appear in its chapter. 


\begin{tabular}{|c|c|c|}
\hline Chapters & ILOs Verbs using Synonyms & Weight of Chapters \\
\hline 1 & 10 & 25 \\
\hline 2 & 9 & 27 \\
\hline 3 & 23 & 2 \\
\hline 4 & 10 & 26 \\
\hline 5 & 14 & 15 \\
\hline 6 & 21 & 3 \\
\hline 7 & 24 & 1 \\
\hline 8 & 15 & 14 \\
\hline 9 & 18 & 7 \\
\hline 10 & 19 & 5 \\
\hline 11 & 13 & 16 \\
\hline 12 & 17 & 9 \\
\hline 13 & 11 & 24 \\
\hline 14 & 13 & 17 \\
\hline 15 & 16 & 10 \\
\hline 16 & 18 & 8 \\
\hline 17 & 21 & 4 \\
\hline 18 & 12 & 22 \\
\hline 19 & 13 & 18 \\
\hline 20 & 13 & 19 \\
\hline 21 & 16 & 11 \\
\hline 22 & 16 & 12 \\
\hline 23 & 6 & 30 \\
\hline 24 & 19 & 6 \\
\hline 25 & 13 & 20 \\
\hline 26 & 9 & 28 \\
\hline 27 & 7 & 29 \\
\hline 28 & 13 & 21 \\
\hline 29 & 12 & 23 \\
\hline 30 & 1 & 31 \\
\hline 31 & 16 & 13 \\
\hline Total & 438 & 31 \\
\hline
\end{tabular}

According to the second semester, the doctor was teaching Java textbook, it was taught in accordance with the weight of the chapters of the textbook, then the doctor select the chapters from the following table and arranges them according to what was arranged in the book. However, because of the short time of the second semester, only 12 chapters of the textbook were taught there are [3-6-7-9-10-12-1516-17-21-22-24]. The next phase will be measured and valuate the results of this experiment compared to historical data.

\section{E. Explore and Measure KPIs Phase}


Educational KPIs defined as a class of performance measurement that is designed to professionally and efficiently evaluate the achievement of an organization as a whole, an organization's advancement toward its strategic goals, or a specific performance inside the organization's [13].

Based on ISSR vision and the objectives of the total quality management (TQM) to explore the factors that influence the performance of the institution academic program and students' success, after analysis chapters of the textbook we has been explored KPIs for students, and for the academic program (courses) and measure these indicators, KPIs results for this method provide a better result than the previous year. KPIs suitable for institution were explored from the experiments are:

1. Academic program assessment: by using keyword extraction to measure the course according to the ILOs Domains (Knowledge and understanding, intellectual skills, and professional skills).

2. Student success Rate: by measuring percentage of students who successfully complete a credit Java course with a grade of $\mathrm{C}$ or better upon the first attempt.

The advantage of this method is that it detects performance indicators by automatic processing for documents instead of use manual method like a questionnaire, and gives recommendations bSefore taught the course, unlike other studies that analyzed the exam questions to discover the performance of academic program and student success rate. Unfortunately, the result is after the students' examination and implementation of the curriculum.

\section{Methodology Evaluation}

From the above discussion, it is clear that extracting keywords of ILOs in ISSR from learning objectives of Java textbook using keyword extraction technique make us explore and measure two KPI shows in following in Table 2:

Table 2. An Adapted List of Exploring KPIs and their Results

\begin{tabular}{|c|c|c|c|}
\hline KPIs & Description & $\mathbf{2 0 1 5 - 2 0 1 6}$ & $\mathbf{2 0 1 6 - 2 0 1 7}$ \\
\hline Student Success Rate & $\begin{array}{c}\text { Percentage of students who successfully } \\
\text { complete a credit Java course with a grade of } \\
\text { C or better upon the first attempt }\end{array}$ & $\% 75$ & $77.27 \%$ \\
\hline $\begin{array}{c}\text { Academic Program } \\
\text { Assessment }\end{array}$ & Course measures & Not measured & $52.05479452 \%$ \\
\hline
\end{tabular}

KPIs are explored and measured based on principles of analysis learning objectives in textbook according to intended learning outcomes for ISSR. Our Trends consist of current level and last year performances. So the current level is performance of the year of (2016-2017) and historical performance of (2015-2016) shown in Table 2.

The proposed model concerns HEIs performance measurement based on two principal criteria: students, and academic program (courses). The criteria are designed to help institution use an integrated approach to explore institution performance that results in Positioning of institutional growth and competitive advantage, according the courses which were selected with their ILOs and the success rate of student. 
Good performance of HEIs is reflected by the growth in institution results which are demonstrated by current level compared to historical performances.

\section{Conclusion}

This work proposed a new model to explore and measure KPIs in HEIs using keyword extraction method for extracting main features from unstructured data (textbook). An approach based on term frequency technique was developed for this purpose. The approach was tested and results showed that, it can be applied for any course. Exploring KPIs depending on analysis learning objectives of textbook in higher education that achieved intended learning outcomes for the institution. Because the teacher can't teach the full course in one semester, it may force him to choose some chapters of the textbook randomly due to the personal experience without indicating for the selection of those chapters which may lead to less the performance of students and institutions. The results showed that using the method we can explore two KPIs student success sate and academic program assessment and can measure indicators and get better results.

\section{References}

1. F. Azma, "Qualitative Indicators for the evaluation of universities performance", Procedia-Social and Behavioral Sciences 2, No.2, pp. 5408-541, 2010.

2. M. Badawy, A. El-Aziz, and H. Hefny, "Analysis of learning objectives for higher education textbooks using text mining", In proceeding of Computer Engineering Conference (ICENCO), 12th International, pp. 202-207, IEEE, 2016.

3. J. Caspersen, N. Frølich, and J. Muller, "Higher education learning outcomes-Ambiguity and change in higher education", European Journal of Education 52, No.1, pp. 8-19, 2017.

4. S. Atkinson, "Taxonomy Circles: Visualizing the possibilities of intended learning outcomes", BPP University, 2013.

5. R. Talib, M. Hanif, S. Ayesha, and F. Fatima, "Text Mining: Techniques, Applications and Issues", International Journal of Advanced Computer Science \& Applications1, No.7, pp.414-418, 2016.

6. C. Wu, and R. Chen, "KPIs (Key Performance Indicators) in Taiwan Basic Education", JMER 4, No.8, pp.565-578, 2014.

7. J. Taylor, "Improving performance indicators in higher education: The academics' perspective", Journal of Further and Higher Education 25, No.3, pp.379-393, 2001.

8. K. Suryadi, "Key performance indicators measurement model based on analytic hierarchy process and trend-comparative dimension in higher education institution", International Symposium on the Analytic Hierarchy Process (ISAHP), Vina Del Mar, Chile. Vol. 3. 2007.

9. R. Bai, X. Wang, and J. Liao, "Extract semantic information from wordnet to improve text classification performance", Advances in Computer Science and Information Technology, pp.409420, 2010.

10. J. Kaur, and V.Gupta, "Effective approaches for extraction of keywords", International Journal of Computer Science Issues (IJCSI), pp.144, 2010.

11. C. Zhang, "Automatic keyword extraction from documents using conditional random fields", Journal of Computational Information Systems 4, No. 3, pp.1169-1180, 2008.

12. S. Beliga, "Keyword extraction: a review of methods and approaches", University of Rijeka, Department of Informatics, 2014.

13. M. Alkhaldi, Y. Gadhoum, "Official Use of Key Performance Indicators in the Education Sector: Case of Saudi Arabia", Asia Pacific Institute of Advanced Research (APIAR), Vol.3, 2017. 